\title{
El tratamiento de las colocaciones en la lexicografía española y alemana: estudio contrastivo ${ }^{1}$
}

\author{
Verónica Ferrando \\ IC Berlín \\ veronikaaramo@hotmail.com
}

Resumen: Un lugar común cuando se habla del tratamiento de las colocaciones en las obras lexicográficas parece ser la falta de sistematicidad y de criterios claros a la hora de recoger este tipo de unidades fraseológicas. Sin embargo, cabe señalar que, en los últimos años, el panorama se ha vuelto algo más alentador, al menos en el campo de los diccionarios escolares, donde — siguiendo la línea de la lexicografía didáctica inglesa, más avanzada en este aspecto- se están dando los primeros pasos para tratar las colocaciones de forma más explícita, diferenciada y sistemática.

En el presente artículo analizaremos los procedimientos que utilizan algunos diccionarios escolares del español y del alemán y propondremos otras formas de inclusión. Tras analizar este aspecto, pasaremos revista a los principales diccionarios especializados en el fenómeno de la combinatoria léxica existentes para el español y el alemán. Nos ocuparemos tanto de las obras en papel como de los diccionarios en línea. Nuestro objetivo final no es otro que el de contrastar la evolución de la lexicografía combinatoria del español y del alemán y apuntar futuros desarrollos.

Palabras clave: colocaciones, lexicografía didáctica española y alemana, diccionarios combinatorios, enseñanza de la fraseología.

1 Una primera versión de este trabajo fue presentada en el XIX Congreso de la Asociación Alemana de Hispanistas celebrado en Münster en marzo de 2013. 


\begin{abstract}
A commonplace when talking about the treatment of collocations in lexicographical works seems to be the lack of systematic and clear criteria when it comes to collecting this type of phraseological units. However, it should be noted that, in recent years, the landscape has become somewhat more encouraging, at least in the field of school dictionaries, where -following the line of the English teaching lexicography, more advanced in this aspect- the first steps are being taken to treat collocations more explicitly, systematically, and differentiated.

In this paper we will look at the procedures that use some of the Spanish and German learner's dictionaries and propose other forms of inclusion. After analyzing this aspect, we will inspect the main dictionaries specializing in the phenomenon of the lexical combinatory relationships existing for Spanish and German. We'll cover both the works on paper and online dictionaries. Our ultimate goal is to compare the evolution of combinatorial lexicography in Spanish and German language and point future developments.
\end{abstract}

Key words: Collocations, Spanish and German Learner's Dictionaries, Combinatorial Dictionaries, Phraseology in Language Teaching. 


\section{Introducción}

Según la teoría lexicográfica, uno de los aspectos que caracteriza -o debería caracterizar- a todo diccionario pedagógico o didáctico es el tratamiento pormenorizado de la combinatoria léxica, pues esta constituye uno de los mayores problemas en el aprendizaje de idiomas.

Son diccionarios didácticos aquellos diccionarios que están destinados específicamente a la enseñanza y aprendizaje de una lengua. Se pueden distinguir dos tipos: diccionarios didácticos para nativos y para extranjeros. Al primer tipo pertenecen los diccionarios escolares y al segundo tipo los diccionarios monolingües para extranjeros, también llamados learner's.

Por lo que respecta a los diccionarios didácticos del español, como ya hemos apuntado en trabajos anteriores (Ferrando 2000), las colocaciones reciben un tratamiento deficiente, pues, en primer lugar, no se registran tantas como sería de esperar y, cuando se recogen en el interior de los artículos, generalmente sólo pueden rastrearse en los ejemplos o en el régimen lexemático de la definición, sin que exista un criterio claro de inclusión.

Este hecho también ha sido puesto de relieve más recientemente por Molina (2010) tras analizar los cuatro diccionarios para estudiantes de E/LE existentes hoy en día en el mercado. En orden de aparición, dichos diccionarios son: Diccionario Salamanca de la lengua española (Santillana-Universidad de Salamanca 1996), Diccionario para la enseñanza de la lengua española (Vox-Universidad de Alcalá de Henares 1997), Diccionario de la lengua española para estudiantes de español (Espasa Calpe 2002) y Diccionario de español para extranjeros (SM 2002). Molina concluye que

los diccionarios monolingües de español L2 necesitan una mayor y mejor representación de las colocaciones, puesto que o bien aparecen poco tratadas o bien no existe un tratamiento sistemático que le sea útil al alumno tanto en la comprensión como en la producción, y especialmente en este último aspecto, que es el que en el aprendizaje de colocaciones ofrece mayores problemas al aprendiz. (Molina 2010: 230)

\section{Las colocaciones en el Diccionario Estudio Salamanca}

Una excepción a la asistematicidad señalada por Molina parece ser el Diccionario Estudio Salamanca (Octaedro 2007) —en adelante, DESAL_, un diccionario escolar que recurre al contorno para proporcionar la información colocacional 
sobre las palabras. Así, en la parte introductoria de la obra se proporciona una definición explícita de las colocaciones y se indica cómo van a incluirse en el interior de las entradas:

En cuanto a las colocaciones, debido a que son combinaciones de palabras completamente libres desde el punto de vista de la lengua, pero que presentan ciertas restricciones impuestas por el uso (acariciar una idea, banco de peces, entablar amistad, estallar una guerra, fracasar estrepitosamente, ignorancia supina, pastilla de jabón, etc.), no van marcadas, llevan la categoría gramatical que le corresponda a cada palabra considerada individualmente y damos información acerca de los verbos o sustantivos, por ejemplo, con los que aparece la colocación, bien redactando con ellos la definición o numerándolos en el campo de la información normativa. (p. XXIV)

Veamos, a título de ejemplo, la entrada correspondiente al adjetivo supino. En su tercera acepción se dice lo siguiente:

La ignorancia, la estupidez u otra cualidad negativa son supinas si se tienen en grado extremo: Cada vez que habla de la historia de España, muestra una ignorancia supina sobre ella. (p. 1020)

Este recurso del DESAL de integrar los verbos o sustantivos de una colocación en la redacción de la definición del colocativo está tomada del Collins Cobuild Advanced Learner's Dictionary (HarperCollins 2009).

Nótese que la colocación se ofrece en el colocativo (supino) y no en la base (ignorancia $)^{2}$. Por tanto, dicha información sólo será útil en las tareas de descodificación. Para favorecer la producción, las colocaciones deberían ofrecerse también bajo las bases, puesto que es el elemento que suele conocer el estudiante y del que, por tanto, parte cuando quiere formular una idea.

A pesar de recoger las colocaciones generalmente bajo el colocativo, el DESAL constituye un importante avance por lo que respecta al modo de incluir la información colocacional en el interior de las entradas, ya que es el primer diccionario general del español en adoptar un criterio homogéneo a la hora de ofrecer colocaciones $^{3}$. El gran mérito del DESAL radica en ser el primer diccionario del español

\footnotetext{
2 Como ha indicado Hausmann (1989), las colocaciones son combinaciones orientadas, en el sentido de que uno de los elementos selecciona al otro. El elemento seleccionador es la base y el elemento seleccionado, el colocativo. Estos elementos no tienen el mismo estatus: la base es semánticamente autónoma, mientras que el colocativo añade una caracterización que no modifica la identidad del caracterizado. La base es el elemento caracterizado, y el colocativo, el elemento caracterizador, el cual sólo realiza plenamente su identidad semántica en la colocación; esto es, conjuntamente con la base. En las colocaciones de sustantivo + verbo, de verbo + sustantivo, de sustantivo + adjetivo y de sustantivo + (preposición) + sustantivo, la base es el sustantivo; mientras que en las colocaciones de verbo + adverbio y en las de adverbio + adjetivo, lo son el verbo y el adjetivo respectivamente. 3 Para más detalles, véase Perdiguero (2010: 133-153).
} 
que opta por integrar la información colocacional de las palabras en la definición de las mismas.

Los demás diccionarios didácticos del español suelen indicar el contorno de las palabras mediante recursos tipográficos (entre barras verticales partidas), tal es el caso del Diccionario de la lengua española para estudiantes de español (Espasa 2002), o recurriendo a fórmulas como «referido a». Este último recurso es el que encontramos, por ejemplo, en el Diccionario de español para extranjeros (SM 2002), donde el adjetivo supino en su segunda acepción se define del siguiente modo: «Referido a una cualidad negativa, que es enorme o extraordinaria: una ignorancia supina».

Mucho más sistemáticos en este punto son los diccionarios didácticos de la lengua inglesa, pues, como señalan Götz-Votteler y Herbst (2009: 51-56), suelen destacar la información colocacional en el interior de las entradas mediante el uso de la letra negrita y acompañarla de ejemplos de uso o incluso presentarla en las collocation boxes (recuadros de colocaciones) donde los diferentes colocados aparecen agrupados en función de la categoría gramatical, como sucede en los diccionarios de colocaciones. Esta segunda opción constituye un primer paso hacia la integración de un diccionario de colocaciones en un diccionario general de aprendizaje y la encontramos, por ejemplo, en el Macmillan English Dictionary for Advanced Learners (Rundell 2010).

\section{Las colocaciones en los diccionarios bilingües español-alemán}

Por lo que respecta a los diccionarios bilingües, en un trabajo anterior (Bargalló et alii 1999), analizamos cinco diccionarios de este tipo que combinaban el español con otra lengua moderna (alemán, catalán, italiano, francés e inglés).

En general, observamos una mayor presencia de colocaciones en este tipo de diccionarios que en los monolingües. Ello se debe al hecho de que los diccionarios bilingües ofrecen un enfoque contrastivo de la lengua. Sin embargo, si bien los diccionarios tenían la voluntad de recoger colocaciones, dada la novedad del concepto en la lingüística hispánica en el momento de la compilación de los mismos, estos no las reconocían explícitamente.

Por lo que respecta al alemán, el repertorio objeto de análisis fue el Langenscheidts Handwörterbuch Spanisch (Müller y Haensch 1971). Este diccionario opta por tres procedimientos diferentes a la hora de recoger las colocaciones: 
a) hacerlo de forma explícita, en cuyo caso operan los mismos criterios de lematización, ubicación y marcación que en las locuciones, como puede apreciarse en la entrada de decisión que reproducimos en el anexo (figura 1) donde la combinación tomar una decisión aparece así recogida.

b) incluirlas como ejemplos;

c) presentar el sustantivo de la colocación bajo la entrada del verbo o adjetivo con el que se coloca en letra cursiva y en la lengua de destino (alemán). Cuando se trata de complementos directos van antepuestos a la traducción, como se aprecia en la traducción del verbo afinar (figura 2 del anexo) y cuando se trata de sujetos van pospuestos y entre paréntesis.

Con este último procedimiento se corre el riesgo de confundir Kollokatoren (colocados) y discriminadores semánticos, que aparecen de la misma forma. Este recurso, a pesar de ser poco afortunado, lo seguimos encontrando en los diccionarios actuales. Tal es el caso, por ejemplo, del Langenscheidt Power Wörterbuch Spanisch (Langenscheidt 2008). Este repertorio presenta también los Kollokatoren entre paréntesis, en letra cursiva y en alemán. Sin embargo, cuando son sujeto aparecen delante de la traducción y cuando son complemento detrás, al revés que en el Handwörterbuch (crf. figuras 3 y 4 del anexo).

Los diccionarios de la editorial Pons optan por una solución algo más acertada: incluir los Kollokatoren en letra cursiva sin paréntesis para así evitar confundirlos con los discriminadores semánticos, que van entre paréntesis. El problema es que los Kollokatoren se siguen ofreciendo en alemán y no en español, de manera que el usuario no es plenamente consciente de encontrarse ante una colocación también en español. Puede reconocer la colocación alemana pero no sabe a ciencia cierta cómo es en español. Esta problemática puede apreciarse, por ejemplo, en la siguiente entrada adjetival del Express Wörterbuch Spanisch (Pons 2008):

\section{acalorado [akalo'rađo], acalorada [akalo- 'rađa] 1 Diskussion: hitzig, erregt 2 Person: hitzköpfig}

Mucho más acertada es la solución que encontramos en el diccionario escolar Encuentros (Cornelsen 2011) cuyos destinatarios son estudiantes de 11 a 16 años. Se trata de un repertorio de nueva planta que opta por un sistema novedoso: ofrece los Kollokatoren por duplicado, en español y en alemán. Antes de la traducción aparecen en español y detrás de la misma en alemán, de este modo no hay posibilidad de confundir la información colocacional con otras informaciones. Es un sistema que permite mostrar los casos de equivalencia colocacional entre el español y el alemán tal como puede apreciarse en la entrada del adjetivo acalorado: 
acalorado, acalorada (ambiente, discusión, persona) hitzig (Atmosphäre, Streit, Person)

El diccionario Encuentros incluye unas 15.500 colocaciones bilingües; la mayoría de las cuales corresponden al tipo verbo + objeto. No obstante, hay colocaciones para los seis tipos establecidos por Hausmann (1989), incluso para los menos frecuentes (p. ej. criticar duramente $s_{.} v_{*}$ «duramente»; extremadamente peligroso $\mathrm{s}_{+} \mathrm{V}_{*}$ «extremadamente»).

En algunos casos las colocaciones aparecen como subentradas numeradas. Se opta por esta solución cuando no existe una colocación alemana equivalente. En estos casos, los traductores debían transformar los Kollokatoren en una subentrada numerada. Por ejemplo, en el artículo de la voz echar (figura 5 del anexo), la colocación echar la basura se presenta mediante el sistema de Kollokatoren porque equivale a la colocación alemana den Müll wegwerfen; ahora bien, echar una bronca aparece como subentrada porque la traducción alemana es una locución verbal. El problema es que al incluir las colocaciones como subentradas se corre el riesgo de confundirlas con locuciones (echar raices). El usuario no sabe que se encuentra ante una colocación.

Respecto al tratamiento de las colocaciones en los diccionarios escolares y bilingües actuales, podemos concluir que, si bien se han producido mejoras considerables respecto a finales del siglo pasado, aún queda un largo camino por recorrer para que los repertorios puedan ser equiparables a los learner's del inglés.

\section{Los diccionarios de colocaciones del español ${ }^{4}$}

La lexicografía combinatoria del español ha dado pasos de gigante; en sólo diez años se han publicado cuatro diccionarios combinatorios‡ el Diccionario Euléxico (Boneu 2001) —en adelante DEu-, Redes. Diccionario combinatorio del español contemporáneo (Bosque 2004) —en adelante, Redes -, el Diccionario combinatorio práctico del español contemporáneo (Bosque 2006) -en adelante, Prácticoy el Diccionario de colocaciones del español (Alonso 2004) - en adelante, DiCE. Algunos de ellos (Práctico y DiCE), como veremos más adelante, ofrecen incluso una estructuración semántica de las colocaciones.

El DEu es un diccionario de autor, pues está redactado íntegramente por J. Boneu. Su aparición constituye una total novedad en la lexicografía española, ya que es el primer diccionario del español centrado exclusivamente en el fenómeno

4 Para una descripción más detallada de este tipo de diccionarios véase Ferrando 2012. 
de la combinatoria léxica. Su lemario está integrado únicamente por sustantivos, un total de 1.800, y los lemas son las bases de las colocaciones. Como ya han advertido Haensch y Omeñaca (2004: 70), se trata de un diccionario parcial de colocaciones, pues sólo registra combinaciones de sustantivo + adjetivo, de verbo + sustantivo, de sustantivo + verbo y de verbo + preposición + sustantivo tal como puede apreciarse en la entrada del sustantivo adorno:

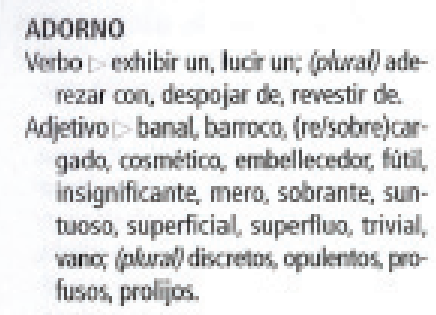

En cuanto a Redes, si bien, como apuntan Battaner y DeCesaris (2006: 400), por el subtítulo podríamos pensar que Redes es otro diccionario combinatorio más, del tipo del The BBI Combinatory Dictionary of English: A Guide to Word Combinations (Benson et alii 1986) o, añadimos nosotros, del Dictionnaire des combinaisons de mots (Le Robert 2007), pues contiene en el título una alusión explícita al fenómeno de la combinatoria léxica, lo cierto es que, como el propio Bosque advierte en su introducción, se trata un diccionario sui generis porque es un diccionario combinatorio pero fundamentado en las relaciones semánticas que se dan entre las palabras. Así pues, no estamos ante un diccionario de colocaciones en sentido estricto ya que, si bien muchas de las combinaciones que ofrece Redes son colocaciones, lo que se específica en cada entrada son las restricciones semánticas que las palabras se imponen unas a otras ${ }^{5}$. En palabras de Bosque:

En Redes se exploran las combinaciones frecuentes tomando como punto de partida los criterios semánticos que permiten agrupar conceptualmente estas voces, y muy especialmente las formas en que los predicados restringen a sus argumentos. (p. LXXXVI)

Lo cierto es que la información combinatoria que ofrece Redes no se muestra de forma explícita en ninguno de los diccionarios de E/LE existentes en el mercado, y esto lo convierte en una obra de consulta indispensable para el alumnado en determinados niveles de aprendizaje.

5 De hecho en Redes se incluyen numerosas expresiones que no suelen considerarse colocaciones (dinero negro) y se omiten otras que sí lo son (sacar la basura). 
Mucho más accesible para los estudiantes de E/LE es, sin lugar a dudas, Práctico ya que está concebido como un diccionario de uso y con el fin de hacerlo menos conceptual se han eliminado las clases léxicas ${ }^{6}$.

En el prólogo, Bosque admite que si bien Práctico contiene un gran número de colocaciones, prefiere presentarlo como un diccionario de combinaciones para evitar asumir el riesgo conceptual de que cada combinación contenida en la obra se corresponda exactamente con lo que la fraseología denomina colocación, pues, algunas de las expresiones registradas podrían ser fruto de una asociación semántica, sintáctica o léxica de otra clase (Bosque 2006: XVII). De hecho, Alonso Ramos ha señalado respecto a Práctico que «muchas de las combinaciones que ahí aparecen nada tienen que ver con las colocaciones» (2010:65); tal es el caso de la combinación adoptar un niño, que es un ejemplo de combinatoria libre frente a otras combinaciones como adoptar una solución o adoptar una medida que también están en Práctico y que sí son colocaciones (Alonso Ramos 2010).

Lo cierto es que el hecho de optar por la denominación de diccionario combinatorio, antes que de colocaciones, se trata de una decisión que, como muy acertadamente ha advertido Model (2007), se debe más a cuestiones terminológicas que al carácter de la obra. Estamos asimismo de acuerdo con este estudioso alemán en que el diccionario hace bien en no limitarse a recoger únicamente las colocaciones evidentes y, en aras de dar facilidades al usuario, incluir también todas aquellas combinaciones problemáticas en la producción de textos, independientemente de cuál sea su clasificación fraseológica.

La obra contiene alrededor de 14.000 entradas. Si bien la mayoría de los lemas son nominales, cuenta también con numerosos lemas adjetivales, verbales y adverbiales, los cuales, como ya señala Bosque en la presentación ( $\mathrm{p}$. XIX) y como nosotros hemos podido constatar mediante el análisis de los diferentes diccionarios combinatorios, son poco frecuentes en los repertorios de colocaciones de otras lenguas.

En cuanto al DiCE, este forma parte de un proyecto de investigación cuyo objetivo es proporcionar recursos en línea para el aprendizaje de las colocaciones. La obra está organizada en campos semánticos. Por el momento su nomenclatura se limita a 219 nombres de sentimiento para los que recoge un total de 20.000 relaciones léxicas (paradigmáticas y sintagmáticas).

Respecto la clasificación semántica a la que hacíamos alusión más arriba, es preciso señalar que en Práctico estamos ante un clasificación semántica implícita mientras que en el DiCE dicha clasificación se explicita mediante glosas y funciones léxicas (abreviaturas latinas). Así por ejemplo, en la sección adjetival de la

6 Para un estudio comparativo de Redes y Práctico véase Barrios (2007). 
entrada reparo 1 del $\mathrm{DiCE}$ (reproducida en la figura 6 del anexo) se nos ofrece un listado de tres colocaciones sustantivo adjetivales (grandes reparos y las sinónimas minimo reparo y pequeño reparo) agrupadas bajo dos glosas o funciones léxicas diferentes (grande/Magn y pequeño/Anti Magn).

La clasificación semántica explícita es la que convierte a un diccionario combinatorio en una herramienta realmente codificadora (permite un acceso onomasiológico a la información). Es necesario indicar en este punto que el $\mathrm{DiCE}$ «juega con ventaja» porque se trata de un diccionario en línea y, por tanto, está libre de las limitaciones espaciales a las que están sometidos los otros tres diccionarios, que son obras en papel. Además, no es sólo un diccionario de colocaciones sino que va mucho más allá. Se trata de una herramienta multifuncional, creada a imagen y semejanza del Dictionnaire d'apprentissage du français langue étrangère ou seconde (Verlinde et alii 2003) —en adelante, DAFLES_, pues el DiCE, como en el caso del leximat francés, integra diferentes recursos léxicos para el (auto) aprendizaje de las colocaciones. Así, aparte de la interfaz de acceso al cuerpo del diccionario, incluye un componente de consultas avanzadas, una herramienta de ayuda a la redacción así como un módulo de actividades didácticas. Nótese que se trata de unas aplicaciones que los otros diccionarios combinatorios analizados no ofrecen.

\section{Los diccionarios de colocaciones del alemán}

El alemán dispone de un diccionario de colocaciones propiamente dicho sólo desde 2011, año de publicación del Wörterbuch der Kollokationen im Deutschen (Quasthoff 2011). La obra de Quasthoff es un diccionario en papel que contiene 3.200 lemas (sustantivos, verbos y adjetivos) y en el que las colocaciones aparecen recogidas bajo la base.

Es un diccionario que agrupa los colocativos por categoría, como puede apreciarse en la entrada de Reise (viaje): 


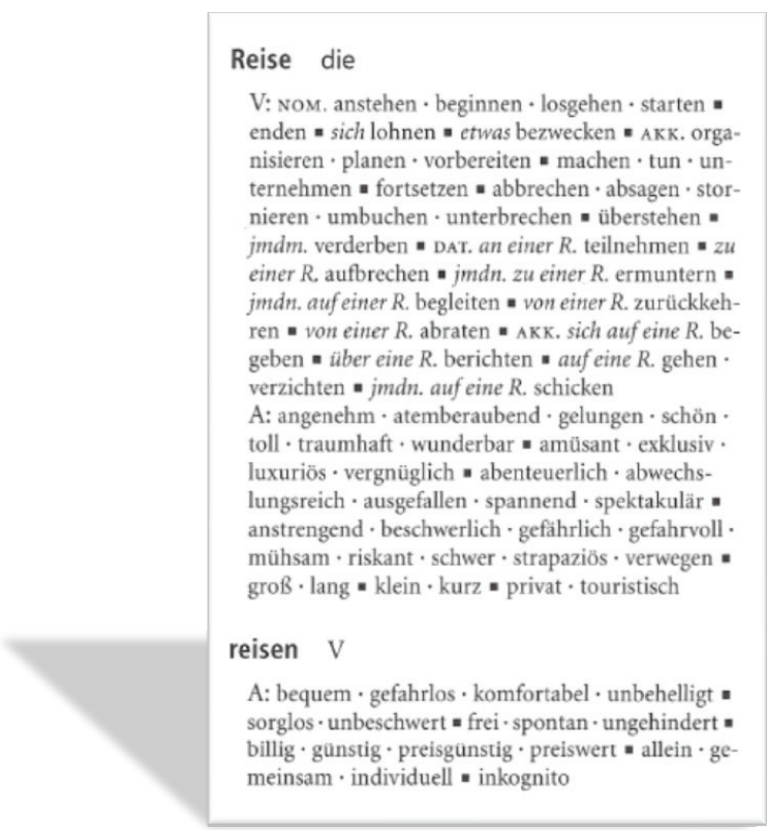

Y dentro de cada grupo categorial encontramos subgrupos combinatorios separados por un cuadratín. En el caso de los verbos, los distintos subgrupos se establecen en función del caso que corresponde al lema en la combinación (que se indica mediante abreviaturas en mayúscula: NOM., AKK. y DAT.) y en función del significado de los verbos. Así, por ejemplo, el primer subgrupo combinatorio incluye los verbos con el significado de 'inicio' que tienen al lema como sujeto (anstehen, beginnen, losgehen, starten). Sin embargo, nótese que, como en el caso de Práctico, se echa en falta una clasificación semántica explícita.

Al igual que el español, el alemán también dispone, si bien desde hace muy poco tiempo, de un diccionario de colocaciones en línea: el Kollokationenwörterbuch. Typische und gebräuchliche Wortverbindungen des Deutschen - en adelante, Kollokationenwörterbuch - proyecto lexicográfico dirigido por Dr. Annelies Häcki Buhofer de la Universidad de Basel ${ }^{7}$. El Kollokationenwörterbuch es un diccionario de colocaciones del alemán (hablado en Alemania, Austria y Suiza) para estudiantes de nivel avanzado y está basado en corpus.

El diccionario se encuentra en fase avanzada de redacción; por el momento registra unas 20.000 colocaciones correspondientes a 400 entradas (aproximadamente una quinta parte de la extensión del diccionario una vez esté terminado). $\mathrm{Al}$ igual que en el $\mathrm{DiCE}$, los lemas son las bases de las colocaciones y estas aparecen agrupadas en función de su categoría gramatical y del significado. En el

7 Se puede consultar en <http://colloc.germa.unibas.ch/web/suche/>. Está previsto que en un futuro cuente también con una versión en papel. 
caso de los colocativos verbales, siempre que es posible, se ofrece una clasificación semántica explícita (al principio de cada subgrupo a modo de superíndice en color verde) como se puede observar en la entrada de Katze (gato):

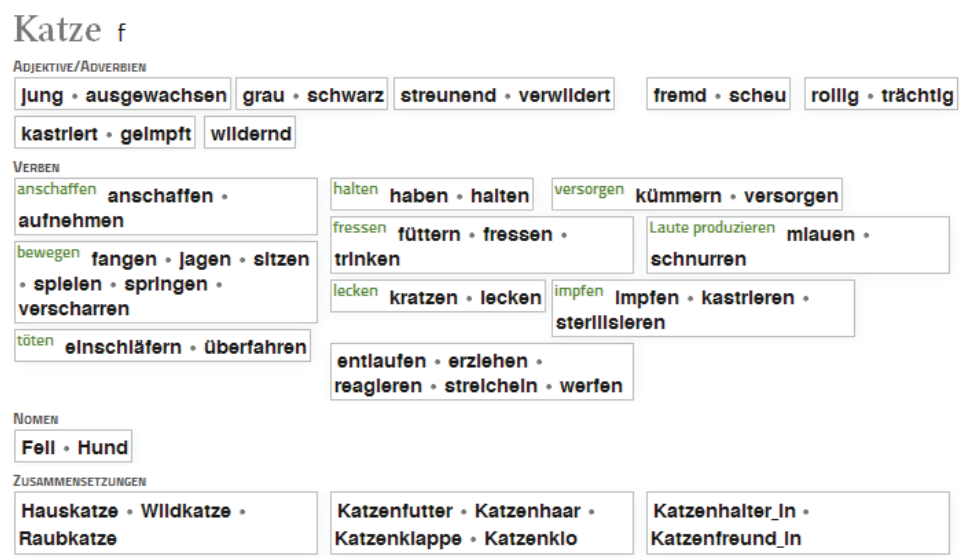

Por otra parte, cabe señalar que este diccionario presenta dos importantes innovaciones frente a los anteriores:

a) Ofrece la posibilidad de encontrar una colocación a partir de cualquiera de sus elementos o incluso escribiendo en la caja de búsqueda la combinación concreta.

b) Presenta tres modos de consulta diferentes para cada entrada: una forma básica o abreviada (como la reproducida arriba), otra forma expandida y, finalmente, la forma completa. La forma básica se limita a ofrecer los listados de colocativos frente a la expandida que, junto a los colocativos, ofrece ejemplos, indicaciones de uso e informaciones semánticas. En cuanto a la forma completa, tal como su nombre indica, incluye no sólo los colocativos sino también la base de las combinaciones.

A la hora de realizar una consulta, la interfaz del diccionario permite, si el usuario así lo desea, combinar la versión expandida y la completa. La entrada resultante de combinar ambas versiones es la que más se asemeja a la que encontraremos en la futura versión en papel y la que resulta más útil para los estudiantes extranjeros, pues es la más informativa ${ }^{8}$.

Un último aspecto que quisiéramos destacar es que los ejemplos de uso están extraídos de corpus. En los modos de consulta básico y expandido, colocando el cursor sobre cualquiera de los colocativos listados, se abre una ventana que nos permite acceder a la colocación completa, a los ejemplos e informaciones semán-

8 En las figuras 7 y 8 del anexo reproducimos respectivamente las versiones expandida y completa de la sección verbal de la entrada de Katze a fin de que el lector pueda compararlas con la entrada simple. 
ticas - cuando se incluyen-, y a las coocurrencias de los colocados en el corpus. Así, por ejemplo, si en la entrada básica de Katze pasamos el cursor sobre el adjetivo schwarz (negro) se nos da la combinación completa (schwarze Katze) acompañada de un ejemplo en cursiva, de la abreviatura de uso "ge» (gebräuchliche Kollokation/Randbereich = colocación usual/alcance) y de la abreviatura sobre fondo gris «CH. TK» (schweizer Textkorpus = corpus textual suizo):

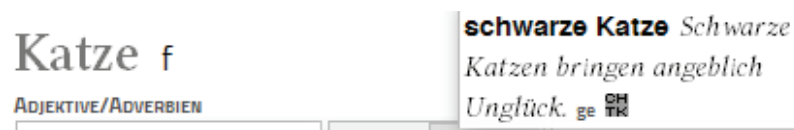

Jung • ausgewachsen grau - schwarz streunend * verwlidert

Clicando sobre esta última abreviatura el programa nos remite a los casos de schwarze Katze - un total de ocho- que figuran en el corpus textual suizo y desde allí se nos permite efectuar nuevas búsquedas (véase figura 9 del anexo).

Está previsto que en un futuro el diccionario incluya enlaces a otros corpus y diccionarios en línea, tanto monolingües como bilingües. Coincidimos con Roth en que la versión en línea del Kollokationenwörterbuch «take the discussion on how collocations dictionaries should be presented online a step further» (2013: 162), conclusión que nos lleva al siguiente apartado del presente artículo.

\section{Nuevos proyectos en curso}

Además de los diccionarios combinatorios descritos en los epígrafes 3 y 4 de este trabajo, existen varios proyectos lexicográficos combinatorios en curso tanto para el alemán como para el español que presentan importantes innovaciones frente a las obras anteriores.

Para el alemán, Christine Konecny del Instituto de Romanística de la Universidad de Innsbruck (Austria) dirige la compilación de un diccionario bilingüe de colocaciones (italiano-alemán) pensado tanto para aprendientes germanohablantes de italiano como para profesores de italiano o alemán. La obra ha sido, pues, concebida como un manual para la enseñanza-aprendizaje de las colocaciones.

Lo más novedoso de este diccionario es que junto a la traducción propiamente dicha de una colocación al alemán, se ofrece su traducción literal para que los usuarios sean conscientes de las diferencias colocacionales. Otro aspecto igualmente novedoso es la disposición de la información colocacional dentro de cada entrada. Las colocaciones aparecen dispuestas en dos columnas diferentes: en la columna izquierda se encuentran las colocaciones italianas y en la derecha la 
traducción al alemán de las mismas, como puede observarse en la entrada modelo Dente (diente) presente en la web del proyecto (<http://www.kollokation.at/>) y cuyo apartado de colocaciones sutantivo verbales reproducimos a continuación:

\begin{tabular}{|c|c|}
\hline il DENTE (m.) & der ZAHN (m.) \\
\hline \multicolumn{2}{|l|}{ Typ 1: Substantiv (Subjekt) + Verb } \\
\hline $\begin{array}{l}\text { il dente dondola (auch: balla, traballa, si muove, [selten]: tentenna) } \\
\text { a qcn. dondola, balla un dente } \\
\text { il dente traballante (auch: che dondola, che balla a qen., che si muove) } \\
\text { Mi balla un dente. }\end{array}$ & $\begin{array}{l}\text { der Zahn wackelt (auch: ist locker) (wörtl.: ,schaukeln"; ballare = tanzen) } \\
\text { jmdm. wackelt ein Zahn } \\
\text { der wackelnde, lockere Zahn } \\
\text { Mir wackelt ein Zahn. }\end{array}$ \\
\hline $\begin{array}{l}\text { - il dente si caria / il dente cariato } \\
\text { Un dente ben curato non si caria spesso. } \\
\text { un dente spunta, sta spuntando (a qen.) (auch: nasce, sta nascendo (a qen.), } \\
\text { compare) } \\
\text { Quando spuntano normalmente i primi denti? }\end{array}$ & $\begin{array}{l}\text { der Zahn fault (auch: wird faul, kariös) / der faule, kariöse Zahn } \\
\text { Ein gut gepflegter Zahn wird meist nicht faul. } \\
\text { - (jmdm.) kommt ein Zahn, ein Zahn bricht durch (wörtl. [in etwa): „die Zahnspitze } \\
\text { [la pumta] taucht auf"; nascere = wörtl.: entstehen; comparire = wörtl.: erscheinen) } \\
\text { Wann kommen normalerweise die ersten Zähne? }\end{array}$ \\
\hline $\begin{array}{l}\text { - (a qen.) cade un dente } \\
\text { Oggi mentre ero nella mensa della scuola mi è caduto un dente da latte. }\end{array}$ & $\begin{array}{l}\text { - (jmdm.) fällt ein Zahn aus } \\
\text { Als ich heute in der Schulkantine war, ist mir ein Milchzahn ausgefallen. }\end{array}$ \\
\hline $\begin{array}{l}\text { (a qen.) si rompe un dente } \\
\text { Due mesi fa mi si è rotto un dente otturato da ormai chissà quanti anni fa. Nel } \\
\text { tentativo di rifare l'otturazione il dentista ha notato che sotto c'era una mova } \\
\text { carie. }\end{array}$ & $\begin{array}{l}\text { (jmdm.) bricht ein Zahn ab } \\
\text { Vor zwei Monaten ist mir ein Zahn abgebrochen, der schon seit wer weiß wie vielen } \\
\text { Jahren plombiert war. Beim Versuch, ihn nochmals zu plombieren, hat der Zahnarzt } \\
\text { bemerkt, dass sich darunter netue Karies gebildet hatte. }\end{array}$ \\
\hline
\end{tabular}

Esta presentación en dos columnas ofrece la posibilidad a los aprendientes que consulten el diccionario de tapar una de las dos columnas para aprender nuevas combinaciones o bien repasar las que ya saben y así comprobar sus progresos.

Los lemas de partida por el momento se limitan a 900-1.100 bases nominales y han sido tomados del vocabulario fundamental del Dizionario di base della lingua italiana de Tulio De Mauro y Gian Giuseppe Moroni (1996). Está previsto que el diccionario sea publicado en formato papel por la editorial Helmut Buske de Hamburgo?.

Otra nueva herramienta en línea centrada en el fenómeno colocacional es el Diccionario de combinaciones léxicas para la enseñanza-aprendizaje de E/LE por niveles (DiCLELE), un diccionario combinatorio del español, temático y por niveles, dirigido a estudiantes de E/LE cuya lengua materna sea el italiano y a sus profesores, del que por el momento hemos desarrollado una muestra completa de un bloque: el dedicado a las combinaciones relacionadas con el ámbito 'viaje ${ }^{10}$.

El DiCLELE ha sido concebido para que sirva no sólo como obra de consulta sino también de instrumento para facilitar el aprendizaje activo por parte de los estudiantes de E/LE y de ayuda para los profesores de español como L2. En este sentido, se han creado dos modos de consulta diferentes: modo profesores y modo

\footnotetext{
9 En un principio su aparición en el mercado estaba prevista para 2013 pero, al menos que sepamos, en el momento de redactar este artículo aún no ha visto la luz.

10 Es una de las veinte categorías que aparecen en el Plan Curricular del Instituto Cervantes, que son las que aparecerán en la versión final del diccionario. En un futuro, confiamos en poder materializar, al menos en parte, los desarrollos aquí esbozados.
} 
estudiantes. El modo profesores está destinado a docentes de E/LE y a los alumnos de nivel avanzado o superior, y en él todas las informaciones aparecen en español.

El modo estudiantes está, en cambio, destinado a los aprendices de nivel inicial o intermedio, y en él, a fin de facilitar la consulta, la interfaz e informaciones como la categoría gramatical, las marcas de registro o las observaciones se ofrecen en italiano ${ }^{11}$.

Además, de cara a potenciar la función del DiCLELE como herramienta de autoaprendizaje está previsto añadir un módulo didáctico así como enlaces a otras páginas electrónicas de utilidad para la enseñanza-aprendizaje de la combinatoria léxica.

Respecto al cuerpo del diccionario, cabe señalar que los lexemas que actúan como lemas son, por norma general, las bases de las colocaciones, como sucede en gran parte de los diccionarios combinatorios existentes hoy en día.

Todos los lemas disponen de uno o más equivalentes en italiano (precedidos de la bandera italiana). En primer lugar se ofrece la traducción contextual, esto es, la que suele corresponder al lema cuando forma parte de las combinaciones registradas; $y$, a continuación, si es necesario, también se ofrece un equivalente de traducción más general (precedido de una bandera italiana más pequeña), como se aprecia en la entrada de alojamiento:

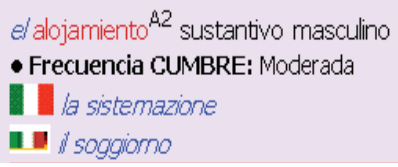

Nótese que los lemas también aparecen acompañados de una indicación sobre la frecuencia de uso (muy alta, alta, notable, moderada, baja o muy baja), información que hemos tomado del Gran diccionario de uso del español actual (SGEL 2001), basado en el corpus lingüístico CUMBRE, de veinte millones de palabras, propiedad de la editorial SGEL.

En la microestructura del DiCLELE se nos ofrecen los elementos que se combinan con cada lema según una triple ordenación. En primer lugar, los colocativos de cada nivel se presentan sobre un fondo de diferente color (rojo para el nivel A, amarillo para el B y verde para el C); después, dentro de cada nivel (A, B o C), los distintos colocativos se agrupan en listados independientes teniendo en cuenta su función sintáctica; y, finalmente, dentro de cada listado, los colocativos se ofrecen semánticamente agrupados dando lugar a subgrupos combinatorios -a modo de

11 Otra diferencia es que en el modo profesores se incluyen informaciones específicas para el docente como la frecuencia de uso de los lemas o una caja de consulta para poder recuperar las combinaciones a partir del colocativo. 
ejemplo, en la figura 10 del anexo reproducimos la entrada de autobús del modo profesores.

En cuanto a la ordenación de los colocativos dentro de cada listado, los colocativos semánticamente relacionados aparecen bajo un mismo subgrupo combinatorio y dispuestos en orden alfabético. Cada grupo de sentido se presenta precedido de un descriptor semántico realzado tipográficamente (en letra mayúscula de color azul y entre corchetes) cuya función es explicitar el significado de la serie. Esta solución permite al usuario encontrar de forma fácil y rápida el colocativo que mejor se ajusta a la idea que desea expresar.

Para satisfacer las necesidades de los estudiantes italohablantes de español, en la microestructura se incluye la traducción al italiano (precedida del símbolo «=» y en un tamaño de letra más pequeño) de las combinaciones registradas, prestando especial atención a los casos que puedan dar pie a falsos amigos colocacionales, los cuales se marcan mediante un símbolo de peligro, como podemos apreciar en la traducción de la colocación sustantivo verbal tomar baños de mar recogida en la entrada de baño:

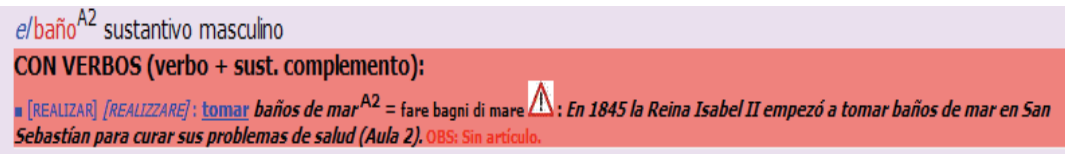

El DiCLELE español-italiano, una vez esté terminado, podrá servir como base para la elaboración de diccionarios combinatorios en línea para los aprendices de otras nacionalidades ${ }^{12}$.

\section{Conclusiones}

La característica más importante de los futuros repertorios de colocaciones analizados en el epígrafe anterior es el estar confeccionados para satisfacer las necesidades de un tipo muy concreto de usuarios. Esto coincide con los nuevos aires en la enseñanza de segundas lenguas.

Tal como ha advertido Concha Maldonado (2012:172), en los últimos tiempos se está empezando a cuestionar la validez del concepto de E/LE por ser demasiado abarcador y se propone hablar de español para italianos, español para japoneses, español para hablantes de alemán, etc.; esto es, se reivindica una enseñanza de español distinta en función de la lengua materna de los alumnos. En este sentido, deberíamos poder contar en un futuro con otros muchos dicciona-

12 Para una descripción más detallada véase Ferrando 2012 (tesis doctoral). 
rios, combinatorios o no, que tengan en cuenta las necesidades concretas de los diferentes tipos de estudiantes de segundas lenguas. Con el presente artículo y más especialmente con nuestro proyecto DiCLELE esperamos estar allanando el camino para que eso sea posible.

\section{Referencias bibliográficas}

Alonso Ramos, M. (2010). «No importa si la llamas o no colocación, descríbela». En: C. Mellado et alii (eds.) (2010). Nuevas perspectivas de la fraseología del S. XXI. Berlin. Frank \& Timme: 55-80.

Bargalló Escrivá, M. et alii (1999). «Unidades fraseológicas y diccionarios bilingües». En: J. FERnÁNDEZ et alii (eds.) (1999). Lingüística para el siglo XXI (Actas del III Congreso de Lingüística General celebrado en Salamanca, los días 23, 24 y 25 de marzo de 1998). Salamanca. Universidad de Salamanca: 247-256.

Barrios Rodríguez, M. (2007). «Diccionarios combinatorios del español: diferencias y semejanzas». RedELE, 11 .

Battaner, P. y J. DeCesaris (2006). «A New Kind of Dictionary: REDES. Diccionario combinatorio del español contemporáneo». En: E. Corino; C. Marello y C. Onesti (eds.) (2006). Proceedings XII EURALEX International Congress. Alessandria. Edizioni dell'Orso: 399-408.

[BBI] Benson, M.; E. Benson y R. Ilson (1986). The BBI Combinatory Dictionary of English: A Guide to Word Combinations. Amsterdam/Philadelphia: John Benjamins.

[DAFLES] Verlinde, S. et alii (2003). Dictionnaire d'apprentissage du français langue étrangère ou seconde. Disponible en: <http://www.kuleuven.ac.be/ dafles $>$ [Consulta: diciembre de 2011].

De Tulio, M. y G. Morini (1996). Dizionario di base della lingua italiana. Torino: Paravia.

[DESAL] VV.AA. (2007). Diccionario Estudio Salamanca. Salamanca: Octaedro. [DEu] Boneu, J. (2001). Diccionario euléxico. Barcelona: Juventud.

[DiCE] Alonso Ramos, M. (2004). Diccionario de Colocaciones del Español [en línea]. La Coruña: Universidad de La Coruña. Disponible en: <http://wwwdicesp.com/> [Consulta: 20 noviembre de 2013].

Ferrando Aramo, V. (2000). «Apuntes sobre el tratamiento de las colocaciones en los diccionarios didácticos del español». En: F. J. Ruíz de Mendoza (coord.) (2000). Panorama actual de la lingüistica aplicada: conocimiento, procesamiento y uso del lenguaje (Actas del XVI Congreso de la Asociación Españo- 
la de Lingüística Aplicada celebrado en Logroño, en abril de 1998). Logroño. Universidad de La Rioja: vol. II.

- (2012). Aspectos teóricos y metodológicos para la compilación de un diccionario combinatorio destinado a estudiantes de E/LE. Disponible en: <http://www. tdx.cat/handle/10803/84025> [Consulta: 20 noviembre de 2013].

Götz-Votteler, K. y T. Herbst (2009). «Innovation in advanced learner's dictionaries of English». Lexicographica, $\mathrm{n}^{\circ} 25,44-66$.

Häскі Buhofer, A. (dir.) (en preparación). Kollokationenwörterbuch. Typische und gebräuchliche Wortverbindungen des Deutschen. Disponible en: <http:// colloc.germa.unibas.ch/web/suche/> [Consulta: 20 noviembre de 2013].

Haensch, G. y C. Omeñaca (2004). Los diccionarios del español en el siglo XXI. Salamanca: Universidad de Salamanca.

Hausmann, F. (1989). «Le dictionnaire de collocations». En: Wörterbücher, Dictionaries, Dictionnaires. Berlin/New York. W. de Gryter: vol. I, 10101019.

Instituto Cervantes (2007). Plan curricular del Instituto Cervantes. Niveles de referencia para el español. Madrid: Instituto Cervantes, Biblioteca Nueva.

Le Robert (2007). Dictionnaire des combinaisons de mots. Paris: Le Robert.

Maldonado González, C. (2012). «Los diccionarios en el mundo ElE: ayer, hoy y mañana (una reflexión desde la propia experiencia)». Revista Internacional de Lenguas Extranjeras, Tarragona, Publicacions URV, No 1: 129-150.

Model, B. (2007). «Diccionario combinatorio práctico del español contemporáneo, Madrid, SM» (reseña). Revista de Lexicografía, A Coruña, Universidad de La Coruña: $193-196$.

Molina Díaz, F. (2010)• «El tratamiento de las colocaciones en los diccionarios monolingües de español L2». En: S. Ruhstaller, y M. D. Gordón (eds.). Diccionario y aprendizaje del español. Bern/Berlin/Bruxelles. Peter Lang: 213-232.

Müller, H. y G. Haensch (eds.) (1971). Langenscheidts Handwörterbuch Spanisch. Berlin/München/Wien/Zürich: Langenscheidt. Reimpr. 1988.

Perdiguero Villarreal, H. (2010). «Logros y retos de los diccionarios del español para extranjeros». En: S. Ruhstaller, y M. D. Gordón (eds.). Diccionario y aprendizaje del español. Bern/Berlin/Bruxelles. Peter Lang: 133-153.

[Práctico] Bosque Muñoz, I. (dir.) (2006). Diccionario combinatorio práctico del español contemporáneo. Madrid: SM.

Quasthoff, U. (2011). Wörterbuch der Kollokationen im Deutschen. Berlin/New York: Walter de Gruyter. 
[Redes] Bosque Muñoz, I. (dir.) (2004). Redes. Diccionario combinatorio del español contemporáneo. Madrid: SM.

Rотн, T. (2013): «Going Online with a German Collocations Dictionary». En: I. Kosem, et alii (eds.) (2013). Electronic lexicography in the 21st century: thinking outside the paper (Proceedings of the eLex 2013 conference, 17-19 October 2013, Tallinn, Estonia). Ljubljana/Tallinn. Trojina, Institute for Applied Slovene Studies/Eesti Keele Instituut: 152-163. Disponible en: <http://eki.ee/elex2013/conf-proceedings/> [Consulta: 20 noviembre de 2013].

Rundell, M. (ed.) (2010). Macmillan English Dictionary for Advanced Learners. Oxford: Macmillan Publishers Limited.

VV.AA. (1996). Diccionario Salamanca de la lengua española. Madrid: Santillana-Universidad de Salamanca.

VV.AA. (1997). Diccionario para la enseñanza de la lengua española. Barcelona: Vox/Universidad de Alcalá de Henares.

VV.AA. (2002). Diccionario de la lengua española para estudiantes de español. Madrid: Espasa Calpe.

VV.AA. (2002). Diccionario de español para extranjeros. Madrid: SM.

VV.AA. (2009). Collins Cobuild Advanced Learner's Dictionary. Glasgow: Harper Collins.

VV.AA. (2008). Langenscheidt Power Wörterbuch Spanisch. Berlin/München/ Wien/Zürich: Langenscheidt.

VV.AA. (2008). Express Wörterbuch Spanisch. Stuttgart: Pons.

VV.AA. (2011). Schulwörterbuch Encuentros. Berlin: Cornelsen Verlag.

VV.AA. (2001). Gran diccionario de uso del español actual. Madrid: SGEL. 


\title{
8. Anexo
}

Figura 1. Entrada deci|sión del Langenscheidts Handwörterbuch Spanisch (Müller y Haensch 1971):

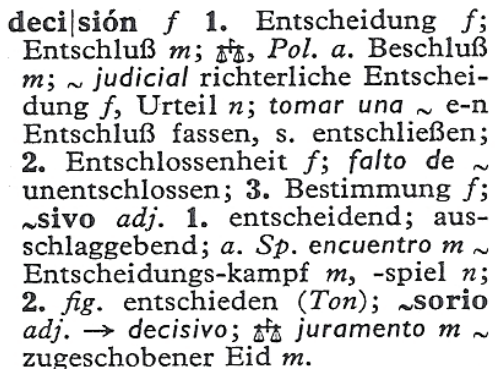

Figura 2. Entrada afina|ción del Langenscheidts Handwörterbuch Spanisch (Müller y Haensch 1971):

\begin{abstract}
afina|ción $f$ Verfeinerung $f$; Stimmen $n$; $\oplus$ Läuterung $f$; Veredelung $f$ der Metalle; damente adv. \& richtig, rein singen usw.; fig. fein, verfeinert; dor $m$ 1. $\delta$ a) (Klavier-)Stimmer $m$; b) Stimmschlüssel $m$; 2. $\oplus$ sid. Abtreiber $m$; udura $f \rightarrow$ afinación; r I. $v / t$. verfeinern, Schliff geben (dat.) (a.fig.); $\oplus$ Metalle läutern; sid.frischen, veredeln; \& Instrumente stimmen; II. $v / i$. tonrein singen (od. spielen); III. $v / r$. se feiner werden.
\end{abstract}

Figura 3. Entrada afinar del Langenscheidt Power Wörterbuch Spanisch (Langenscheidt 2008):

\author{
afinar [afi'nar] 1. stimmen (Musikin- \\ strument) 2. verfeinern
}

Figura 4. Entrada adusto/-a del Langenscheidt Power Wörterbuch Spanisch (Langenscheidt 2008)ः

\author{
adusto, -a [a'đusto, -a] 1. (Person) \\ mürrisch 2. (Gegend) rau
}


Figura 5. Entrada echar del Scbulwörterbuch Encuentros (Cornelsen 2011):

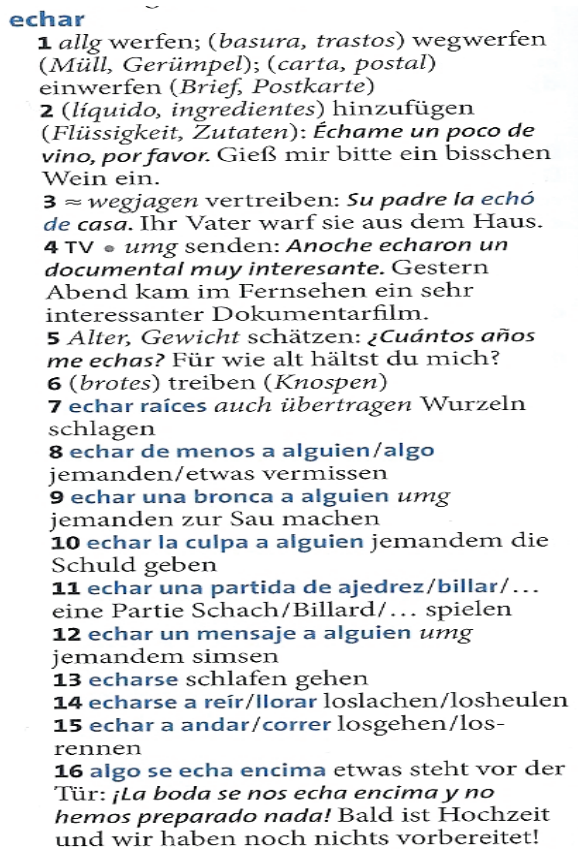

\section{Figura 6. Listado colocaciones sustantivo adjetivales de la entrada reparo 1 del DiCE (Alonso Ramos 2004):}

Encontradas 3 colocaciones

reparo 1 (comunicación o situación)
$\begin{aligned} & \text { Colocaciones } \\ & \text { ver todas, atributo de los participantes, reparo + adjetivo, verbo + reparo, reparo + verbo, nombre de reparo }\end{aligned}$

$[+]$ Desplegar todo $[-]$ Contraer todo Ocultar funciones léxicas ordenar por frecuencia

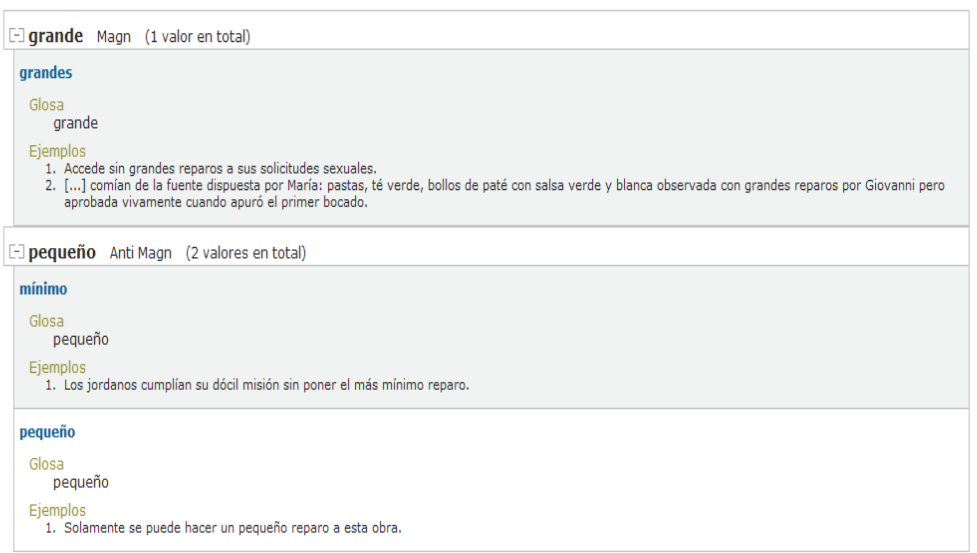


Figura 7. Entrada en su versión expandida de Katze en el Kollokationenwörterbuch;

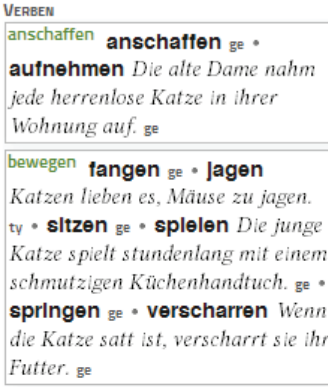

\begin{tabular}{|l|}
\hline halten haben $\mathrm{ge} \cdot$ halten Diese \\
Wohnung ist zu klein, um darin \\
eine Katze zu halten. ty \\
\hline Laute produzieren mlauen ty \\
schnurren ty \\
\hline lecken kratzen ty $\cdot$ lecken Die \\
Katze legt sich an die Sonne und \\
leckt sich ausgiebig ihr Fell. ge \\
\hline $\begin{array}{l}\text { entlauten ty } \cdot \text { erzlehen } \\
\text { reagleren ty } \cdot \text { strelcheln } \\
\text { werfen ty }\end{array}$ \\
\hline
\end{tabular}

\begin{tabular}{|c|}
\hline $\begin{array}{l}\text { versorgen kümmern ge • } \\
\text { versorgen }{ }_{\text {ge }}\end{array}$ \\
\hline $\begin{array}{l}\text { fressen füttern Die Kinder füttern } \\
\text { Nachbars Katze mit } \\
\text { Käsestückchen. ty • fressen ty • } \\
\text { trinken ge }\end{array}$ \\
\hline $\begin{array}{l}\text { impfen Impfen ty } \cdot \text { kastrleren ty } \\
\text { sterlllsleren ge }\end{array}$ \\
\hline $\begin{array}{l}\text { tōten elnschlăfern Der Tierarzt } \\
\text { musste die verletzte Katze } \\
\text { einschläfern. ty } \cdot \text { aberfahren ge }\end{array}$ \\
\hline
\end{tabular}

\section{Figura 8. Entrada en su versión completa de Katze} en el Kollokationenwörterbuch:

\begin{tabular}{l} 
VEABEN \\
anschaffen sIch eIne Katze \\
anschaffen - eIne Katze \\
aufnehmen \\
\hline Laute produzieren eIne Katze mlaut \\
- elne Katze schnurrt \\
\hline lecken elne Katze kratzt an etw. \\
elne Katze leckt sich (das Fell) \\
\hline töten elne Katze elnschlăfern. \\
elne Katze aberfahren \\
\hline
\end{tabular}

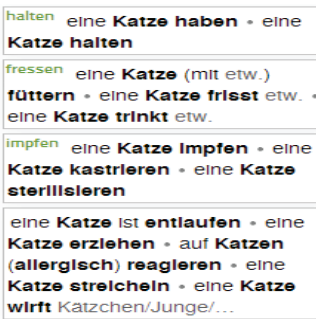

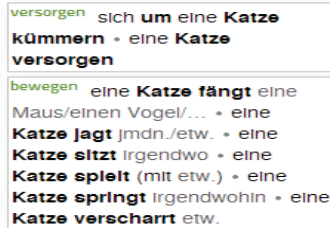

Figura 9. Coocurrencias de schwarz + Katze en el schweizer Textkorpus (Kollokationenwörterbuch):

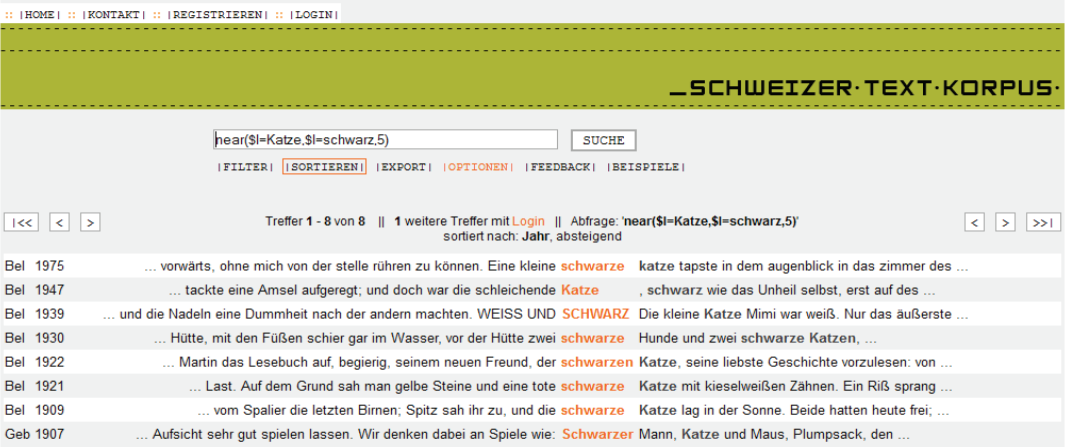




\section{Figura 10. Entrada de autobús del modo profesores en el DiCLELE}

(Ferrando 2012):

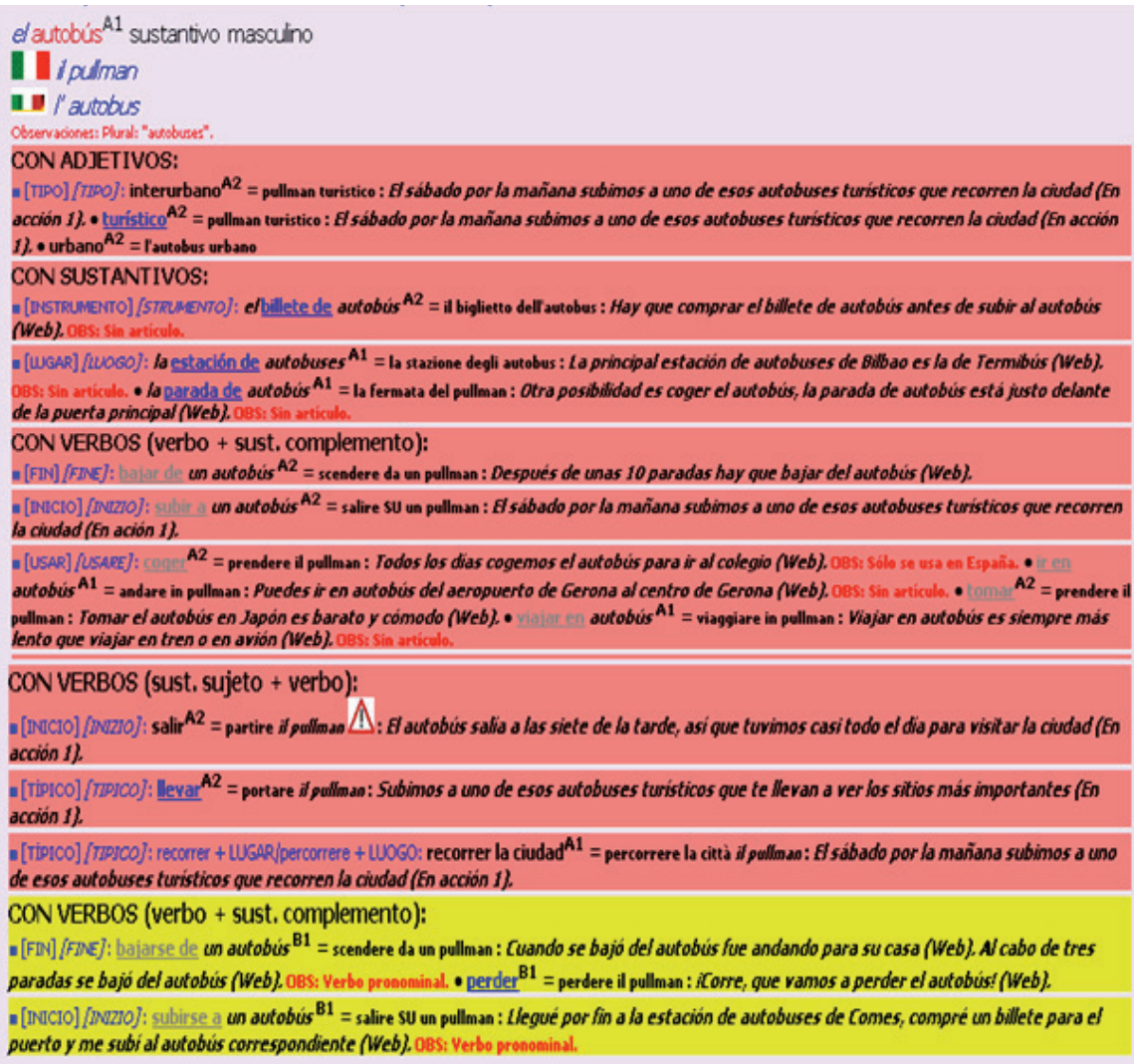

